\title{
Correction to: Mechanism analysis of combined acid-and-ethanol shock on Oenococcus oeni using RNA-Seq
}

\author{
Hongyu Zhao ${ }^{1} \cdot$ Longxiang Liu $^{1,2} \cdot$ Lin Yuan $^{1} \cdot \mathrm{Kai} \mathrm{Hu}^{1} \cdot$ Shuai Peng ${ }^{1} \cdot \mathrm{Hua}^{\mathrm{Li}} \mathrm{i}^{1,3,4,5} \cdot$ Hua Wang $^{1,3,4,5}$
}

Published online: 13 August 2020

○) Springer-Verlag GmbH Germany, part of Springer Nature 2020

\section{Correction to: European Food Research and Technology https://doi.org/10.1007/s00217-020-03520-0}

In the original publication, there are errors in three sentence and reference number, the corrected presentation as follows:

In Abstract section (Page 1637): "producing ATP" should be deleted,

In Energy and substrate supplying section (Page 1643): "or that the ATP is mainly produced by F0F1-ATPase, which was induced by $\triangle \mathrm{pH}$ (pHex-pHin)" this sentence and the reference 43 should be deleted,

In Conclusion section (Page 1644): "and producing ATP" should be deleted,
The original article can be found online at https://doi.org/10.1007/ s00217-020-03520-0.

Hua Li

lihuawine@nwafu.edu.cn

$\triangle$ Hua Wang

wanghua@nwafu.edu.cn

1 College of Enology, Northwest A and F University, 22 Xinong Road, Yangling 712100, Shaanxi, China

2 Shandong Engineering and Technology Research Center for Wild Plant Resources Development and Application of Yellow River Delta, Binzhou, China

3 Shaanxi Engineering Research Center for Viti-Viniculture, Yangling, Shaanxi, China

4 China Wine Industry Technology Institute, Yinchuan, Ningxia, China

5 Engineering Research Center for Viti-Viniculture, National Forestry and Grassland Administration, Yangling, Shaanxi, China
The references number " $44,45,46,47$ " should be replace by " $43,44,45,46$ ", respectively, in the text and References section (Page 1643, 1644, and 1646). 